\title{
EVALUATION OF MULTIPLE LINEAR REGRESSION MODEL TO OBTAIN DBH OF TREES USING DATA FROM A LIGHTWEIGHT LASER SCANNING SYSTEM ON-BOARD A UAV
}

\author{
M.V.Machado ${ }^{1, *}$,A.M.G. Tommaselli ${ }^{2}$, V.M. Tachibana ${ }^{3}$, R. P. Martins-Neto ${ }^{1}$, M.B. Campos ${ }^{1}$ \\ ${ }^{1}$ Post Graduate Program in Cartographic Science, São Paulo State University (UNESP), Presidente Prudente-SP, Brazil \\ (marcelavmachado, rorai.neto, marianaa.bcampos)@gmail.com \\ ${ }^{2}$ Dept. of Cartography, São Paulo State University (UNESP), Presidente Prudente -SP, Brazil \\ a.tommaselli@unesp.br \\ ${ }^{3}$ Dept. of Statistics, São Paulo State University (UNESP), Presidente Prudente -SP, Brazil \\ vilma.tachibana@unesp.br
}

\section{Commission VI, WG VI/4}

KEY WORDS: Airborne Laser Scanning, Unmanned Aerial Vehicles, multiple linear regression, forest application.

\begin{abstract}
:
Vegetation mapping requires information about trees and underlying vegetation to ensure proper management of the urban and forest environments. This information can be obtained using remote sensors. For instance, lightweight systems composed of Unmanned Aerial Vehicles (UAVs) as a platform, low-cost laser units and the recent miniaturized navigation sensors (positioning and orientation) have become a very feasible and flexible alternative. Low-cost UAV-ALS systems usually provide centimetric accuracy in altimetry, according to flight data configuration and quality of observations. This paper presents a feasibility study of a lightweight ALS system on-board a UAV to estimate the diameters at breast height (DBH) of urban trees using LiDAR data and linear regression model. A mathematical model correlating the crown diameter and height of the tree to estimate the DBH was developed based on a linear regression with stepwise method. The stepwise linear regression method enables the addition and the removal of predictor variables through statistical tests. The tree samples were separated in two classes (A and B), according to the diametric distribution. These sample classes were used to define two linear regression models. The regression models that best fit the samples achieved an $\mathrm{R}^{2}$ adj value above $94 \%$ for class A and B, which demonstrates the closeness between the samples and the developed mathematical models. The quality control of the proposed regression models was performed comparing the DBH values estimated and directly measured (reference). DBH of the trees were estimated with an average discrepancy of $8.7 \mathrm{~cm}$.
\end{abstract}

\section{INTRODUCTION}

Diameter at Breast Height (DBH) is an important dendrometric variable that contributes to several forest and urban vegetation monitoring tasks, such as tree growth, volume, basal area and biomass estimation, for forest inventory and management (Kershaw et al., 2016). DBH estimation is usually performed manually using tapelines, calipers and other instruments. This manual data collection is labour-intensive, time consuming, costly and more disposed to gross error. As an alternative, DBH can be estimated by indirect measurements, for instance, using remote sensors and mobile platforms. Several approaches could be developed combining different sensors, platforms and data processing methodologies. This work focused on the use of LiDAR data from Airborne Laser Scanning (ALS) systems for DBH estimation.

ALS is the sensor most used in the acquisition of forest data. The penetrability of the laser beam associated with the record of multiple returns makes the ALS systems an important tool for data acquisition and 3D representation. However, conventional airborne ALS are still high-cost systems, especially when used as an operational tool for forest management. Recently, lightweight low-cost sensors have available on the market and have motivated much research in the development of low-cost ALS.
Unmanned Aerial Vehicles (UAVs) can be combined to miniaturized laser scanning and navigation sensors, resulting in fast, flexible, low-cost ALS systems. Research focusing on the estimation of DBH using low-cost ALS on-board a UAV can be singled out (Jaakkola et al., 2010; Wallace et al., 2012 and Guo et al. 2017).

Jaakkola et al. (2010) presented a low-cost mini-UAV-based laser scanning system (FGI Sensei) composed of a GPS/IMU positioning system, two laser scanners (Ibeo LUX and Sick LMS151), a CCD camera, a spectrometer and a thermal camera. These authors proposed an automatic estimation of the DBH based on the random forest algorithm (nonparametric regression) using UAV-LiDAR data. The DBH was estimated with an RMSE (Root Mean Square Error) of $2.1 \mathrm{~cm}$. Wallace et al. (2012) carried out a data acquisition using a UAV-ALS system composed by an Ibeo LUX laser unit, MEMS based IMU, GPS receiver and a video camera on-board an Oktocopter UAV platform. In this work, dendrometric variables of individual trees in a sparse environment were estimated from a UAV-ALS point cloud with centimetric accuracy $(0.34 \mathrm{~m})$. Tree heights were predicted with a precision of $0.26 \mathrm{~m}$ using an ALS point cloud with a density of 8 points per $\mathrm{m}^{2}$. More recently, Guo et al. (2017) evaluated the correlation between aboveground biomass (AGB) and DBH, using a linear regression method and LiDAR data. The LiDAR

\footnotetext{
* Corresponding author
} 
data was also obtained from a low-cost UAV-ALS system using a Velodyne Puck VLP-16 laser scanner. The Canopy Height Model (CHM) of a mangrove forest derived from the UAV-ALS was generated with 1-meter accuracy.

These related works mentioned above show that UAV-ALS approaches can provide a satisfactory $3 \mathrm{D}$ reconstruction of trees, which can be regarded as an alternative to the conventional ALS for forest management. In this regard, this paper presents a feasibility study of a lightweight ALS approach on-board a UAV to estimate tree height, crown diameter and DBH from a CHM derived from the UAV-LiDAR data. The proposed UAV-ALS is detailed in Section 2. The trees DBH were estimated with a mathematical model based on a linear regression that uses stepwise method to correlate DBH with the Crown diameter $(\mathrm{Cd})$ and Total height $(\mathrm{Ht})$ of the trees (Section 3). Experimental assessment and results of the feasibility study are presented and discussed in section 4 .

\section{UAV-LIDAR SYSTEM AND MATHEMATICAL MODEL}

The lightweight UAV-ALS system used in this paper is composed of an Ibeo LUX laser unit (Figure 1.b), a IMU/GNSS SPAN-IGM-S1 (Inertial Measurement Unit /Global Navigation Satellite System) with a NovAtel-FlexPak6 GNSS receiver (Figure 1.c) and two microcomputers (Raspberry Pi, Figure 1.d) integrated with an octopter UAV (SX8 model). The use of the Ibeo LUX laser unit was motivated by its compact structure $(164.5 \times 93 \times 88 \mathrm{~mm})$ with a weight less than $1 \mathrm{~kg}$, and the measurement-range from 0.3 to $200 \mathrm{~m}$. The nominal ranging resolution of this laser unit is $4 \mathrm{~cm}$ in terrestrial application. However, considering UAV platforms, an average altimetric accuracy of $10 \mathrm{~cm}$ is achieved, as presented by Jaakkola et al., (2010), Wallace et al., (2012) and Torres and Tommaselli (2018).

The UAV-LiDAR devices were synchronized using a postprocessing synchronization (offline) with a precision of $0.7 \mathrm{~ms}$. The process of point cloud generation was implemented in the C++ language, developed by Torres and Tommaselli (2018). Figure 1.a illustrates the proposed lightweight UAV-ALS system.

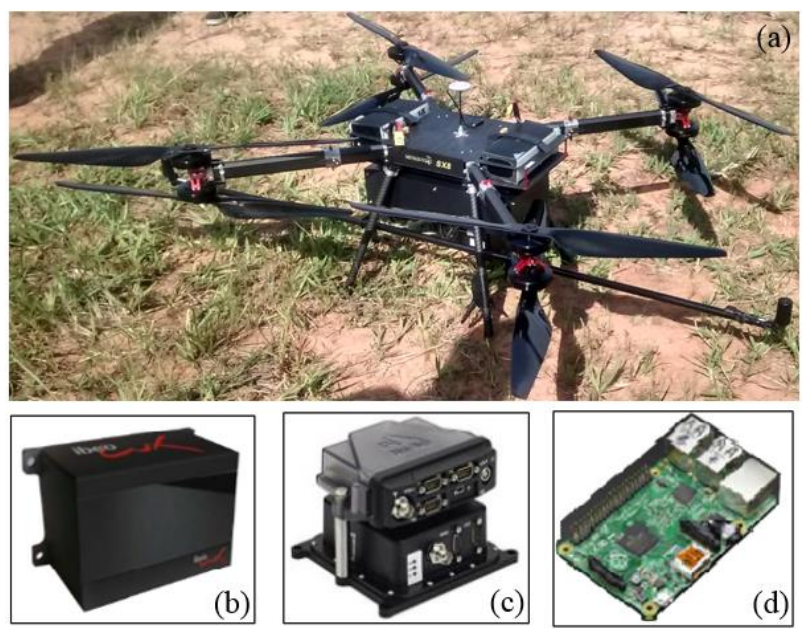

Figure 1. Lightweight low-cost UAV-ALS system: (a) UAVALS system; (b) Ibeo LUX unit; (c) navigation system and (d) recording unit (Raspberry Pi).

The laser mathematical model used to generate the UAV-ALS point cloud is presented in Equation 1 (El-Sheimy et al., 2005, adapted by Torres and Tommaselli, 2018).

$$
r_{i}^{g}=r_{G N S S}^{g}(t)+R_{I M U}^{g}(t) r_{U L}^{I M U}+R_{I M U}^{g}(t) R_{U L}^{I M U} R_{D E}^{U L}(t) \rho_{I}
$$

Where: $r_{i}^{g}$ is the vector of ground coordinates of point $i$; $r_{G N S S}^{g}(t)$ is the vector of the ground coordinates of the GNSS antenna at instant $t$, reduced to the IMU coordinate system; $R_{I M U}^{g}(t)$ is the rotation matrix relating the ground and IMU coordinate systems at instant $t$, derived after processing the GNSS and IMU data;

$r_{U L}^{I M U}$ corresponds to the offset between the laser unit and IMU origin (lever-arm);

$R_{D E}^{U L}$ is the rotation matrix relating the laser unit coordinates system and the laser emitting devices (mirror scans angles) at instant $t$ (as the laser unit used in this research scans four horizontal layers, the rotation matrix is expressed as a function of two angles $\beta$ and $\theta$ );

$\rho_{I}$ is the vector of the coordinates of point $i$ expressed in the emitting device reference system.

$R_{U L}^{I M U}$ is the rotation matrix that relates the laser unit and IMU coordinate systems as a function of the approximate angles directly measured $(\Delta \kappa, \Delta \varphi, \Delta \omega)$. The initial values $R_{U L}^{I M U}$ elements are given as: $\Delta \kappa=-90^{\circ}, \Delta \varphi=-90^{\circ}$, and $\Delta \omega=0^{\circ}$.

The LiDAR point cloud obtained with the proposed UAV-ALS system can be used to generate Digital Terrain Models (DTM), Digital Superficies Model (DSM), CHM for forest and other applications. The CHM enables the estimation of tree parameters, such as total tree height, crown diameter and the indirect prediction of DBH using statistical mathematical models, such as regression-based methods. A stepwise linear regression algorithm is an option to develop a mathematical model, selecting a set of optimal predictor variables to estimate a response variable. The methodology to predict DBH from individual trees using a stepwise linear regression algorithm is described in the next section.

\section{A METHODOLOGY FOR DBH ESTIMATION USING LINEAR REGRESSION}

According to Hair et al. (1998), multivariate analysis techniques enable simultaneous analysis of multiple measures from individuals or objects under investigation, helping users in decision-making. Multivariate measurements can be modelled with multiple linear regression models which consider one response variable for two or more predictor variables (Johnson and Wichern, 2007). Therefore, the linear regression method enables the development of mathematical models to predict response variables (dependent) considering the relationship with predictor variables (independent or explanatory).

Multiple linear regression models are extensively applied in environmental sciences. McGarigal et al. (2013) mentioned that multivariate analysis techniques can reflect the multidimensional and multivariate nature of ecological systems considering the statistical significance of the relationship between the predictor and response variables. Therefore, tree parameters are widely modeled using multivariate-based methods. For instance, the relations obtained by linear models enabled the estimation of dependent variables that are difficult to obtain with direct measurements, such as volume, biomass and crown diameter, from independent variables such as total height, DBH and their respective transformations (Kozak, 1970; Payandeh, 1983).

In this regard, this section presents a methodology to define a mathematical model to estimate indirectly the DBH of individual trees in an urban area. This methodology is based on a linear regression applied to jackknife samples (Efron and Tibshirani, 
1993), in which the subset of predictor variables used in the final equation can be found, e.g., by a stepwise procedure (Johnson and Wichern, 2007). The stepwise method was used to determine a mathematical model that correlates DBH with the crown diameter and total height of the tree in urban areas. The proposed methodology can be summarized in two steps: the arrangement of the samples in classes considering the diametric distribution (DBH size) and mathematical model determination combining a delete-one jackknife approach with the stepwise method.

Two main challenges are to be noted in tree mapping in urban areas. First, urban areas are generally composed of heterogenous tree species, which hinders linear regression modelling due to the absence of a standard behavior. Therefore, the set of trees in the test area can be separated in classes, for instance, in function of the DBH dimension or the tree species, generating different linear models for each class of trees in the test area. Second, urban areas are composed of small samples of trees, especially compared to forest areas. Thus, a delete-one jackknife approach was evaluated for establishing statistical models and to enhance predictions for data sets with few observations (here $<10$ ). The jackknife focuses on the sample that leaves out one observation at a time. A set of the observations $(n-1)$, called training set, will be used to build a model by stepwise procedure and validating the analysis of the observation left out (cross-validation). This process is performed iteratively, in which the ith jackknife sample consists of the data set with the ith observation removed (Efron and Tibshirani, 1993). The set of jackknife sample that provides the smallest error in the cross-validation is used to define the mathematical model.

The stepwise method is applied for each set of jackknife sample to select the predictor variables for the mathematical model that best represents the response variable. In a model with only one predictor variable, $\beta_{0}$ is the point where the line intersects the $y$ axis (intercept) and $\beta_{1}$ is the angular coefficient (slope) that indicates the variation of the mean of $Y$ according to the increase of a unit from the $\mathrm{X}$ variable. More details about the stepwise method can be found in Johnson and Wichern (2007). The multiple regression model is described by Equation 2 .

$$
Y=\beta_{0}+\beta_{1} X_{1}+\cdots+\beta_{p} X_{p}+\varepsilon
$$

Where: $Y$ is the value of the response variable;

$X_{j}$ is the value of the predictor variable with $j$ values ranging from 1 to $\mathrm{p}$;

$\beta_{j}$ is the regression coefficients or parameters of the mathematical model $(j=1,2, \ldots, p)$;

$\varepsilon$ is the error or residual in the adjustment of the linear regression model.

The stepwise method generates different sets of parameters. The model that best explains the response variable is chosen according to a statistic criterion, for instance, considering the adjusted coefficient of multiple determination $\left(\mathrm{R}^{2} \mathrm{adj}\right)$ and the $\mathrm{P}$ value. $R^{2}$ adj was used to explain the percentage of adjustment of the variables to the model, while, the P-value is used to evaluate the significance of a predictor variable, considering a level of significance $(\alpha)$ as reference. A significance level $(\alpha)$ of 0.05 was considered to estimate the significance of the variables that will compose the mathematical model to estimate the DBH. Therefore, a variable with a P-value higher than $\alpha$ is considered a predictor variable in the model, otherwise, the variable is removed from the model. In addition to this analysis, regression coefficients were also evaluated, which describe the size and direction of the relation between a predictor variable and the residuals of the observations.

After this process, linear regression models were generated for each class of trees considering the combination of predictor variables to estimate each tree DBH (response variable). The total height, the crown diameter and their variations are used as predictor variables to develop the linear regression mathematical model. These predictor variables were indirectly measured using the LiDAR data obtained with the proposed UAV-ALS system. LiDAR data acquisition and processing (Section 4.1), the estimation of predictor variations (Section 4.2) and the evaluation of the linear regression mathematical model (Section 4.3) are presented in Section 4.

\section{EXPERIMENTS AND RESULTS}

\subsection{Data Acquisition and Processing}

The data set was collected with a UAV-ALS system in an urban area located at São Paulo State University campus (UNESP), in Presidente Prudente (22 $\left.07^{\prime} \mathrm{S}, 51^{\circ} 24^{\prime} \mathrm{W}\right)$, Brazil. The flight height used to collect the data set was approximately 35 meters, with a flight speed of $4 \mathrm{~m} / \mathrm{s}$. The lateral overlap between flight strips ranges from $60 \%$ to $80 \%$, in a north-south direction. Two strips were used to generate the point cloud (Figure 2). The point cloud accuracy was $0.28 \mathrm{~m}$ in planimetry and $0.1 \mathrm{~m}$ in altimetry. The average density of the point cloud was 11.65 points per $\mathrm{m}^{2}$.

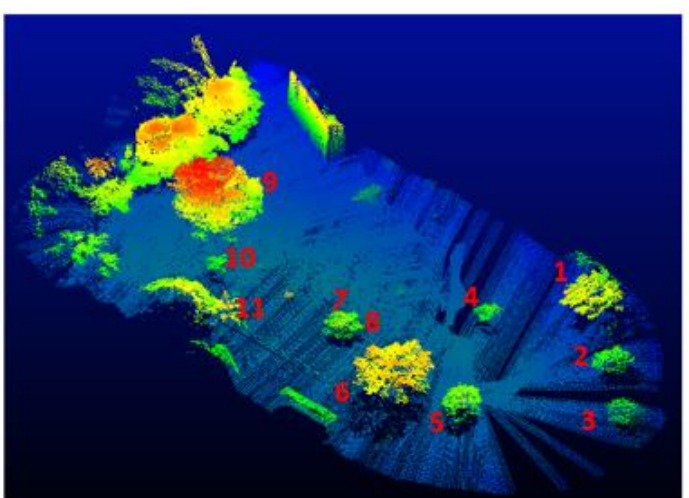

(a)

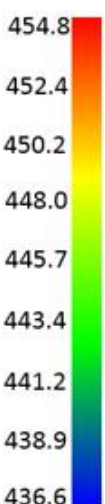

[m]

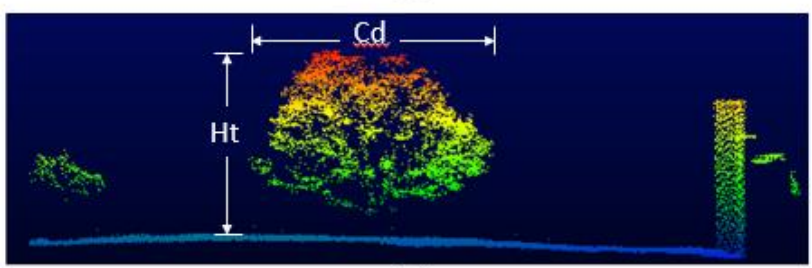

(b)
Figure 2. UAV- LiDAR point cloud: (a) three-dimensional view of the point cloud and (b) lateral profile showing mainly the structure of a tree.

\subsection{Estimation of Predictor Variables and Linear Regression Model Determination}

The UAV-LiDAR data was used to generate a CHM, enabling the indirect estimation of the total heights and the crown diameters of the trees mapped in the test area. The CHM was generated in a two-step process: first, the generation of DTM and DSM, and then the estimation of the difference between them, which results in a CHM. The generation of DTM and DSM were 
performed using LASTools software, which are presented in Figure 3.a and Figure 3.b, respectively.

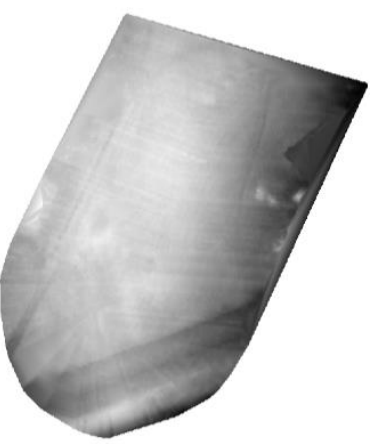

(a)

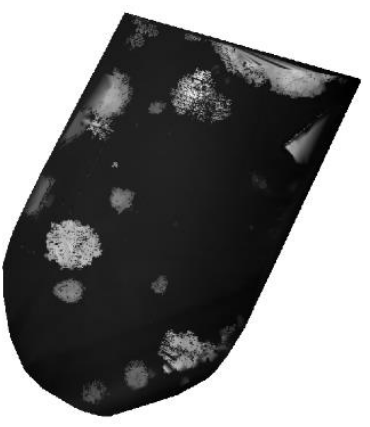

(b)
Figure 3. Digital Terrain Model and Digital Surface Model used for CHM generation.

The difference between DSM and DTM was performed using the free software Quantum Gis 3.2.3. The height of the CHM obtained ranges from 0 to $15.31 \mathrm{~m}$. The maximum height is compatible with the highest tree identified in the test area. Predictor variables (total heights and crown diameters of the trees) were then extracted from the CHM. The height of the canopy was measured in the CHM considering the central axis of each tree. The crown diameter was obtained calculating the mean of the largest and smallest axis of the tree.

CHM assessment was carried out comparing the heights obtained from the CHM with the tree heights directly measured in a field survey. The direct measurements were taken using a tacheometer (Imaging Total Station GPT-7005i, Figure 4), providing reference height values with centimetric accuracy. The statistical mean, standard deviation, and RMSE of the discrepancies between the tree heights estimated from the CHM and the reference data (tacheometry) were $0.08 \mathrm{~m}, 0.236 \mathrm{~m}$ and $0.236 \mathrm{~m}$, respectively. The accuracy of the estimated tree heights can be regarded as compatible with the results obtained by Wallace et al. (2012), which reached $0.26 \mathrm{~m}$. This result can be explained by the similarity between the point clouds, in terms of altimetric accuracy $(10 \mathrm{~cm}$ and $15 \mathrm{~cm})$ and density $\left(11\right.$ and 8 points per $\left.\mathrm{m}^{2}\right)$. The CHM accuracy can be improved by increasing the point cloud density, as also discussed by Wallace et al. (2012).

The predictor variables obtained from the CHM $(\mathrm{Ht}, \mathrm{Cd}, \mathrm{ln} \mathrm{Ht}$, ln $\left.\mathrm{Cd}, \mathrm{Ht}^{2}, \mathrm{Cd}^{2}\right)$ were used to define the linear regression model that best fit the data set. The trees composing the data set are illustrated in Figure 4. The non-homogeneous features in the same area are a predominant behaviour in urban areas with a diversity of species. This species diversity, with different sizes of crown, trunk diameter and total height, hinders the generation of a single linear regression model. A preliminary assessment was carried out considering the complete data set to fit the linear regression model, resulting in high errors in $\mathrm{DBH}$ estimation. The set of trees in the test area was therefore separated into two classes as a function of the DBH dimension. Class A with diameters ranging from $0.10 \mathrm{~m}$ to $0.3 \mathrm{~m}$, and Class $\mathrm{B}$ with diameters higher than $0.3 \mathrm{~m}$, resulting in two linear regression models.
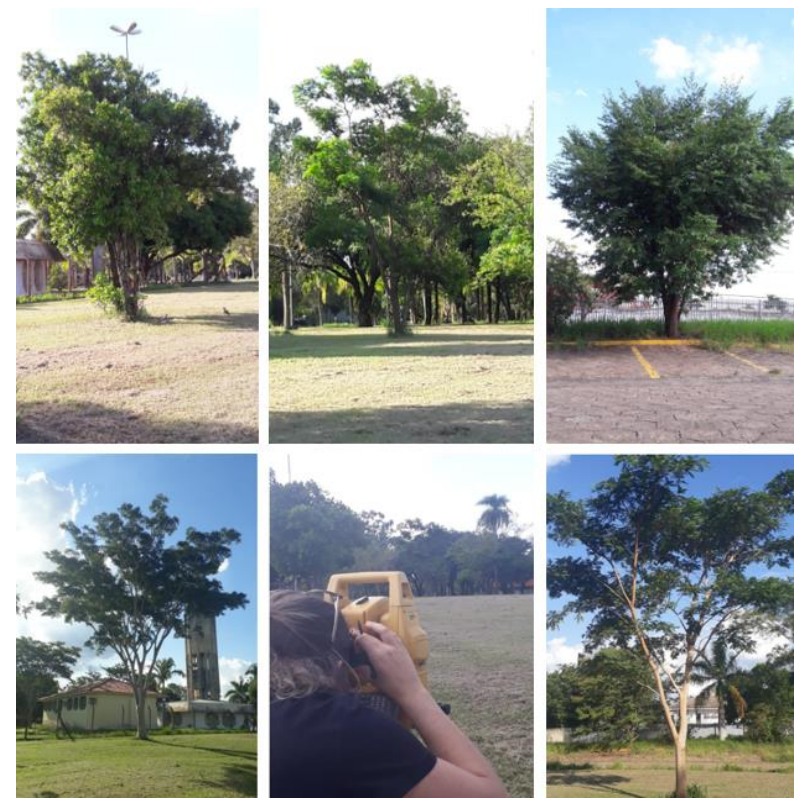

Figure 4. Features of the trees belonging to the test area.

Six sets of jackknife samples, with four observations each, were assessed based on the error estimated in the cross-validation for class A and class B. The training set used to define the final mathematical model by the stepwise method is presented in Table 3. The predictor variables $\mathrm{Cd}, \mathrm{Ht}$ and their variations were assessed by the stepwise method. According to the p-value presented in Table 1, the predictor variable Neperian logarithm of $\mathrm{Cd}(\ln \mathrm{Cd})$ was considered significant for class A and class B for a significant level $(\alpha)$ of 0.05 . The regression mathematical models that best fit the jackknife samples (training set) for class $\mathrm{A}$ and class $\mathrm{B}$ are presented in Equation 3 and Equation 4, respectively.

$$
\begin{gathered}
D B H=32.19-7.15 \ln C d \\
D B H=-104.3+90.5 \ln C d
\end{gathered}
$$

The estimated coefficients (Coef.) presented in Table 1 describe the size and direction of the relation between predictor and response variable. The negative coefficient indicates that a smaller crown diameter of the tree will result in a higher DBH for class $\mathrm{A}$. In class $\mathrm{B}$, the significant variables (ln $\mathrm{Cd}$ ) describe a more stable relation between $\mathrm{DBH}$ and crown diameter, considering more mature trees with a greater diameter.

\begin{tabular}{|c|c|c|c|}
\hline CLASS & Term & Coef. & P-value \\
\hline \multirow{2}{*}{$\mathrm{A}$} & Constant & 32.19 & 0.003 \\
& Ln Cd & -7.15 & 0.017 \\
\hline \multirow{2}{*}{$\mathrm{B}$} & Constant & -104.3 & 0.053 \\
& Ln Cd & 90.5 & 0.017 \\
\hline
\end{tabular}

Table 1. P-value and regression coefficients of the adjusted model for classes $\mathrm{A}$ and $\mathrm{B}$.

Table 2 presents the precision (Std) and the adjusted coefficient of multiple determination $\left(R^{2}\right.$ adj) of the mathematical model obtained in the stepwise process. The precision (Std) of the regression models presented in Equations 3 and 4 were $0.89 \mathrm{~cm}$ and $10.45 \mathrm{~cm}$, respectively. The result for class $\mathrm{B}$ can be associated to the high variability of the DBH of trees. Class A presented more similar tree diameters, ranging between $13 \mathrm{~cm}$ and $26 \mathrm{~cm}$, which was better modeled by the linear regression than the samples from class B. The DBH in class B ranges 
between $0.35 \mathrm{~m}$ to $1.41 \mathrm{~m}$. The values of $\mathrm{R}^{2}$ adj shows that the predictor variable describes more than $94 \%$ of the variability of the DBH model in class A and class B.

\begin{tabular}{|c|c|c|}
\hline Class & Std & $\mathrm{R}^{2}(\operatorname{adj})$ \\
\hline A & 0.89 & $94.92 \%$ \\
B & 10.45 & $94.89 \%$ \\
\hline
\end{tabular}

$\alpha$ to enter $=0.05 ; \alpha$ to remove $=0.05$

Table 2. Precision (Std) and the adjusted coefficient of multiple determination $\left(\mathrm{R}^{2}\right.$ adj) resulting from the stepwise method for class A and class B.

Table 3 shows the jackknife samples used to define the regression mathematical model for class A and class B. The residuals $(\varepsilon)$ of the model were computed considering the difference between the DBH estimated with the proposed mathematical models and the sample values directly measured. The average of the residuals is close to zero $(0.01 \mathrm{~cm})$, showing the absence of systematic errors.

\begin{tabular}{|c|c|c|c|c|}
\hline \multirow{2}{*}{ Class } & \multirow{2}{*}{ Tree } & \multicolumn{3}{|c|}{ DBH $(\mathrm{cm})$} \\
\cline { 3 - 5 } & & $\begin{array}{c}\text { Sample } \\
\text { values }\end{array}$ & Estimated & $\varepsilon$ \\
\hline & 4 & 22.09 & 21.23 & 0.86 \\
A & 6 & 13.31 & 13.11 & 0.20 \\
& 10 & 21.26 & 21.44 & -0.18 \\
& 11 & 18.49 & 19.38 & -0.89 \\
\hline & 2 & 87.00 & 74.54 & 12.46 \\
B & 3 & 50.13 & 57.26 & -7.13 \\
& 7 & 38.99 & 41.22 & -2.23 \\
& 9 & 141.62 & 144.37 & -2.75 \\
\hline
\end{tabular}

Table 3. Samples of trees used to define the linear mathematical models to estimate DBH for class A and class B.

\subsection{Assessment of the Linear Regression Model}

The assessment of the regression models was carried out comparing the estimated DBH with direct measurements performed in a field survey (in loco). The reference values were obtained with a centimetric accuracy using a calliper. The DBH measurements used in the quality control were not used for the regression mathematical model estimation (independently observed). The models were generated with four observations and validated with one observation, due to the low number of samples. DBH was estimated with a difference of $8.84 \mathrm{~cm}$ for Class A and $8.47 \mathrm{~cm}$ for Class B, considering the discrepancies between the estimated and reference $\mathrm{DBH}$ values, as presented in Table 4.

These results using a data set collected by lightweight ALSsystem can be compared to results that estimate DBH at plot level using conventional ALS data in areas with heterogeneous trees. Many studies used LiDAR data from conventional ALS to measure predictor variables, such as total height and crown diameter, aiming at an estimation of DBH (response variable) with a linear regression model. For instance, Ibanez et al. (2016) used linear regression models with LiDAR data to estimate DBH at plot levels of tropical forests. The $\mathrm{r}^{2}$ of the adjusted model was $0.70 ; 0.83$ and 0.72 for the three plots tested with $5 \times 5 \mathrm{~m}^{2}, 10 \times 10$ $\mathrm{m}^{2}$ and $2 \times 20 \mathrm{~m}^{3}$ respectively. Accuracies (RMSE) of $6.08 \mathrm{~cm}$; $2.63 \mathrm{~cm}$ and $2.60 \mathrm{~cm}$ were obtained for these three plots. DBH estimation in plot level experiments usually presents better results than experiments considering individual trees. The proposed methodology also estimated DBH with centimetric accuracy.
The discrepancy presented by class A (tree diameter $<0.3$ ) and class B (tree diameter $>0.3 \mathrm{~m}$ ) can be considered compatible. Both classes achieved a discrepancy between the estimated DBH and the reference of approximately $8 \mathrm{~cm}$. These discrepancies can be associated with tree maturity. The DBH estimation is a challenge for trees that are still developing. Furthermore, the test area is composed of urban trees from different species and heterogeneous features, as presented in Figure 4. Homogeneous forests present a similar tree structure, enabling better results, which is the case in the boreal forest presented by Jaakkola et al. (2010).

\begin{tabular}{|c|c|c|c|c|}
\hline \multirow{2}{*}{ Class } & \multirow{2}{*}{ Tree } & \multicolumn{3}{|c|}{ DBH $(\mathrm{cm})$} \\
\cline { 3 - 5 } & & Reference & Estimated & $\Delta_{\text {DBH }}$ \\
\hline A & 5 & 26.29 & 17.45 & 8.84 \\
B & 8 & 43.16 & 34.69 & 8.47 \\
\hline
\end{tabular}

Table 4. The linear mathematical model assessment by crossvalidation for class $\mathrm{A}$ and class $\mathrm{B}$.

\section{CONCLUSIONS}

This paper presented a feasibility study on the use of data generated by a lightweight ALS system on-board a UAV to estimate tree heights and DBH from a CHM derived from the point cloud. Tree heights were estimated with an accuracy of 0.23 $\mathrm{m}$, which reflects the consistency of the CHM generated by the proposed ALS system. These results are similar to the accuracy presented by Wallace et al. (2012). The DBH variable was estimated by linear regression, according to two classes of tree determined in function of diametric distribution. Class A presented a discrepancy of $8.84 \mathrm{~cm}$ for trees with diameters ranging between $0.1 \mathrm{~m}$ and $0.3 \mathrm{~m}$ and class $B$ present $8.47 \mathrm{~cm}$ for larger diameter trees $(>0.3 \mathrm{~m})$. Despite the species variability and the small sample size in the test area, this work presented preliminary results demonstrating the feasibility of the proposed UAV-ALS system in the estimation of the DBH of individual trees, with centimetric accuracy. In future works, experiments with larger sample areas and with lower flight heights will be performed to improve the results.

\section{ACKNOWLEDGEMENTS}

The authors would like to acknowledge the Coordenação de Aperfeiçoamento de Pessoal de Nível Superior - Brasil (CAPES - Finance Code 001) and the Fundação de Amparo à Pesquisa do Estado de São Paulo (FAPESP - Grant: 2013/50426-4) for their financial support. The authors also acknowledge financial support from ISPRS Foundation.

\section{REFERENCES}

Efron, B and Tibshirani, R. J. (1993). An introduction to the Bootstrap. Boca Raton, Florida, USA.

El-Sheimy, N., Valeo, C., Habib, A. (2005). Digital Terrain Modeling: Acquisition, Manipulation and Applications. Artech House, Norwood, USA.

Guo, Q., Su, Y., Hu, T., Zhao, X., Wu, F., Li, Y., Liu, J., Chen, L., Xu, G., Lin, G., Zheng, Y., Lin,Y., Mi, X., Fei, L., Zheng, Y. (2017). An integrated UAV-borne lidar system for 3D habitat mapping in three forest ecosystems across China. International Journal of Remote Sensing,38(8-10), 2954-2972. doi.org/10.1080/01431161.2017.1285083 
Hair, J. F., Anderson, R. E., Tatham, R. L., Black, W. C. (1998). Multivariate Data Analysis. Upper Saddle River, New Jersey, USA.

Ibanez, C. A. G., Carcellar III, B. G., Paringit, E. C., Argamosa, R. J. L., Faelga, R. A. G., Posilero, M. A. V., Zaragosa, G.P., Dimayacyac, N. A. (2016). Estimating DBH of trees employing multiple linear regression of the best lidar-derived parameter combination automated in python in a natural broadleaf forest in the Philippines. International Archives of the Photogrammetry, Remote Sensing and Spatial Information Sciences, XLI-B8,657662. doi.org/10.5194/isprsarchives-XLI-B8-657-2016.

Jaakkola, A., Hyyppä, J., Kukko, A., Yu, X., Kaartinen, H., Lehtomäki, M., Lin, Y. (2010). A low-cost multi-sensoral mobile mapping system and its feasibility for tree measurements. ISPRS Journal of Photogrammetry and Remote Sensing, 65(6), 514522. doi.org/10.1016/j.isprsjprs.2010.08.002

Johnson, R. A. and Wichern, D. W. (2007). Applied multivariate statistical analysis. Upper Saddle River, New Jersey, USA.

Kershaw Jr, J. A., Ducey, M. J., Beers, T. W., Husch, B. (2016). Forest mensuration. John Wiley and Sons, New Jersey, USA.

Kozak, A. (1970). Methods for ensuring additivity of biomass components by regression analysis. The Forestry Chronicle, 46(5), 402-405.

McGarigal, K., Cushman, S. A., Stafford, S. (2013). Multivariate statistics for wildlife and ecology research. Springer Science \& Business Media, New York, USA.

Payandeh, B. (1983). Some applications of nonlinear regression models in forestry research. The Forestry Chronicle, 59(5), 244248 .

Torres, F. M. and Tommaselli, A. M. G. (2018). A lightweight UAV based laser scanning system for forest application. Bulletin of Geodetic Sciences, 24(3), 318-334. doi.org/ 10.1590/s198221702018000300021

Wallace, L., Lucieer, A., Watson, C., Turner, D. (2012). Development of a UAV-LiDAR system with application to forest inventory. Remote Sensing, 4(6), 1519-1543. doi.org/10.3390/rs4061519 